\title{
ISTORIOGRAFIJA
}

ROBERT MUCHEMBLED, PhD, Professeur émérite

Université Paris XIII, Sorbonne-Paris-Cité

Paris, France

pregledni rad

primljeno: 29. jun 2016.

prihvaćeno: 8. februar 2017.

\section{LA REPRESSION SEXUELLE: UN MOTEUR DU PROGRES OCCIDENTAL, DE 1500 A NOS JOURS?}

ABSTRACT: Dans ce texte, l'auteur examine les évolutions et les répercussions d'une très puissante répression sexuelle qui s'installa au cœur même de la civilisation occidentale vers le milieu du XVIe siècle et ne céda réellement du terrain qu'à partir des années 1960. Producteur d'une tension fondatrice entre la libido de l'individu et les idéaux collectifs, ce processus a constamment développé, durant cette longue période, un puissant effort de sublimation, sous des couverts culturels successifs liés à la religion, aux Lumières, à la médecine du XIXe siècle et au marché capitaliste. De 1960 à nos jours se développa une nouvelle approche de la sexualité occidentale témoignant de mouvement culturels et sociaux profonds.

MOTS CLES: Répression sexuelle, civilisation occidentale, religion, Lumières, médecine, capitalisme, culture, société

Contrairement à Michel Foucault dans le premier volume de son Histoire de la sexualité paru en 1976, je pense qu'une très puissante répression sexuelle s'est installée au cœur même de la civilisation occidentale vers le milieu du XVIe siècle, ne cédant réellement du terrain qu'à partir des années 1960. Producteur d'une tension fondatrice entre la libido de chacun et les idéaux collectifs, le processus a constamment développé un puissant effort de sublimation, durant cette longue période, sous des couverts culturels successifs (religion, Lumières, médecine du XIXe siècle, lois du marché capitaliste).

Sur le socle coercitif fermement établi au XVIIe siècle se sont ensuite imposés en alternance des cycles de libération puis de contrainte, dont le mouvement représente pour moi un facteur explicatif primordial du dynamisme général de l'Europe, parce qu'il crée obstinément le besoin de combler le déséquilibre produit dans les consciences. Car nombre d'individus soumis, volontairement ou non, aux tyrannies de la rigueur morale développent une structure comportementale qui les pousse littéralement en avant, contribuant à porter leurs talents personnels à l'incandescence, dans de multiples secteurs d'activité, tels le prosélytisme religieux, la guerre et la conquête du reste du globe, les activités artistiques ou intellectuelles, le commerce international... 
Utiles pour analyser le thème aux XVIIIe et XIXe siècles, les paradigmes dégagés par Foucault ne s'appliquent ni avant ni après. La situation se révélait très différente aux XVIe et XVIIe siècles, lorsque la répression résultait moins d'une pression morale et légale exercée par des tutelles lointaines que de celle produite par le regard des autres et les codes de comportement locaux. L'archétype dont il dessine les contours est en réalité issu d'un changement fondamental parti des métropoles populeuses, Paris et Londres, vers 1700, pour imposer un rééquilibrage général du système sexuel au profit des adultes mâles, à travers l'affirmation de plus en plus exclusive du lien conjugal.

\section{Les paradigmes de Foucault}

Parfois un peu confuses, les démonstrations de Foucault visent à déplacer l'attention des réalités physiques vers les discours, en prétendant révéler une logorrhée croissante aboutissant à faire du sexe le secret primordial, parce qu'il est réellement la « raison de tout». Son argument central : à partir du XVIII ${ }^{\mathrm{e}}$ siècle naît un bio-pouvoir qui travaille en profondeur le problème de la jonction entre le corps et la population et fait de la sexualité sa cible centrale.

Foucault modifia complètement le plan de la série qu'il annonçait dans le premier tome. Il ouvrait cependant deux pistes intéressantes : 1. l'idée de mystère sexuel ultime, livré au seul confesseur ou plus tard évoqué sur le divan du psychanalyste, paradoxalement véhiculé par une masse grandissante d'écrits consacrés au thème. 2. on ne peut pas parler d'un cycle répressif unique, avec un début et une fin.

Les 4 paradigmes (ensemble de connaissances admises par la communauté scientifique) qu'il identifie contiennent, quoi qu'il en dise, une forte charge répressive : hystérisation du corps de la femme réalisée par la médecine ; pédagogisation du sexe de l'enfant, destinée à endiguer de graves périls, notamment l'onanisme ; socialisation des conduites procréatrices par l'encadrement de la fécondité des couples et l'accentuation de leur «responsabilisation»; psychiatrisation du plaisir pervers, pour mieux identifier les anomalies et les pathologies à soigner.

Ce sont les traits fondamentaux d'une période centrale, les XVIIIe et XIXe siècles, mais ils ne caractérisent pas les deux temps historiques qui l'encadrent. La seule évolution linéaire depuis la Renaissance me parait se situer dans les discours d'enfermement de l'énigme sexuelle au cœur même du Moi, non pas dans les comportements réels, marqués par plusieurs grandes ruptures successives.

\section{Mise en place de la répression sexuelle (XVIe-XVIIe siècles)}

\section{L'ancien modèle paysan}

Les sociétés rurales de l'Europe préindustrielle accordent au mariage chrétien un rôle très différent de celui observé dans les mondes urbains ou parmi les élites, car il s'articule sur une structure relationnelle originale, composée de trois éléments séparés : le groupe des hommes mariés, le groupe des femmes mariées avec les petits, et le groupe des jeunes, composé essentiellement des garçons. 
Le système permet de gérer la sexualité en faisant d'elle le lieu symbolique primordial d'une négociation permanente entre les classes d'âge masculines : les adultes contrôlent ainsi étroitement le potentiel érotique perturbateur des générations montantes en confinant leurs membres dans un long stade d'attente. Rassemblés dans des « royaumes » ou des " abbayes de jeunesse », qu'ils doivent tous rejoindre sous la pression des traditions, ils rongent leur frein à partir de la puberté en attendant d'accéder à la plénitude de l'existence, définie non seulement par la possession de biens et de pouvoirs mais plus encore par le statut matrimonial qui permet de s'insérer dans le cercle des dominants locaux.

En contrepartie, ces bandes juvéniles offrent à leurs membres protection et solidarité, afin de leur permettre de résister aux exigences des géniteurs ou des maîtres, et leur donnent de multiples occasions de défoulement, sous l'œil très compréhensif du reste de la paroisse. Leurs membres sont notamment autorisés à agir comme les gardiens de la sexualité légitime, en imposant des chevauchées parodiques sur un âne aux maris cocus ou en attirant publiquement l'attention sur des amants adultères, par exemple en traçant un chemin végétal entre les deux maisons. Ces droits sont une manière de reconnaître le statut de frustrés des jeunes hommes, car les filles et les femmes mariées du lieu sont étroitement surveillées par leurs pères, leurs frères et leurs parents, qui n'hésitent pas à poursuivre de terribles vengeances contre un amoureux non accepté par eux (le texte littéraire de Roméo et Juliette suit ce schéma).

Les célibataires peuvent cependant profiter de circonstances où la surveillance se relâche, pour courtiser les demoiselles à la veillée ou sous leur fenêtre et avoir avec elles des rapports physiques furtifs, y compris au XVIIe siècle, lorsque le code moral et religieux se renforce. Car leur sexualité est contrainte, mais pas inexistante. Toute proie féminine sans protecteur mâle ou insuffisamment surveillée risque le pire, comme au XVe siècle dans les petites villes du sud-est de la France (Dijon...), où des bandes de "fils à marier " commettent impunément des viols collectifs (Jacques Rossiaud). Les mâles célibataires profitent aussi de toutes les occasions qui s'offrent : épouses insatisfaites, veuves esseulées, prostituées. Et ils s'adonnent probablement à la masturbation. Les sources n'en parlent guère avant les manuels de confession de la fin du XVIe siècle, qui dénoncent énergiquement ce péché. Beaucoup d'historiens bien-pensants ont conclu un peu vite à la rapide efficacité des nouvelles normes en train de s'imposer, notamment en Angleterre sous l'effet du puritanisme culpabilisateur. Mais ces conduites, considérées auparavant comme banales et bien tolérées, survivent. En Somerset, de 1601 à 1660, selon Geoffrey Quaife, il semble que la «masturbation hétérosexuelle mutuelle jusqu'au point de l'éjaculation masculine puisse avoir été l'exutoire sexuel pour un nombre considérable de membres des ordres inférieurs avant le mariage ». Quant à la sodomie et à la bestialité, théoriquement punies de mort par de sévères édits princiers à partir du XVIe siècle, elles ne sont pas véritablement devenues des interdits au village. En France, les procès de ce type concernant des paysans sont rarissimes, ce qui indique probablement l'existence d'une indulgence tacite à ce sujet. Frustrés d'expériences sexuelles régulières, les grands garçons célibataires jusqu'au mariage (25 ans au XVIIe siècle, 30 ans en 1789) n'ont-ils pas la tentation de chercher du plaisir dans les bras des membres des royaumes de jeunesse ? Un autre exutoire leur est largement 
ouvert : la violence sanguinaire contre des semblables de paroisses voisines. Car ces excès sont tolérés par les adultes du lieu, comme par le roi, qui pardonne facilement un meurtrier célibataire : «il faut bien que jeunesse se passe ». Le recul constant de l'âge au mariage attise les frustrations érotiques, car en théorie, toute sexualité hors mariage est un péché et un crime aux yeux de la loi.

Profondément patriarcal, adapté à un monde paysan majoritaire $(80 \%$ de la population ou plus), le système repose sur un pacte tacite entre les générations et sur le principe de permanence des structures sociales. Car les fils devenus pubères ne peuvent accepter leur longue marginalisation que dans l'espoir de prendre un jour la place de ceux qui les précèdent.

\section{La répression sexuelle}

La sublimation est à mes yeux le véritable moteur de la dynamique européenne depuis qu'elle a été intimement liée, voici cinq siècles, à un puissant effort de répression sexuelle dépassant largement le cadre de la morale chrétienne. Le phénomène remonte loin. Les moines s'en étaient faits les champions dès l'origine du christianisme, invitant les prêtres séculiers à les imiter en valorisant le célibat et la continence sexuelle. Il fallut cependant attendre le concile de Trente, en 1563, pour voir appliquer avec une rigueur croissante de telles consignes, alors que des papes, de nombreux évêques et une foule de prêtres catholiques avaient auparavant des enfants. Et ce n'est qu'à partir du moment où les membres du clergé donnèrent un meilleur exemple que ces idées furent adoptées par des cercles laïques actifs de plus en plus larges et gagnèrent une place cruciale dans l'expérience européenne.

À l'époque de la Renaissance, le discours chrétien met en garde contre la volupté, l'idéal étant pour l'homme, et plus encore pour la femme, de s'en passer totalement, de rejoindre un monastère pour faire son salut en niant les impératifs de la chair. Mais les réalités sont très différentes. Si l'Eglise catholique considère le mariage comme indispensable pour tous ceux qui ne peuvent refréner leurs tentations, afin d'éviter la damnation, les pouvoirs locaux se contentent le plus souvent de faire respecter un équilibre tacite fondé sur la surveillance des excès par tous les paroissiens. Malgré le regard des autres (la loi de la honte) et la pratique de vengeances sanguinaires, la sexualité se révèle assez libre, productrice d'un certain nombre de bâtards (Jean-Louis Flandrin).

Un changement radical se produit lentement, entre le milieu du XVIe siècle et la fin du suivant. Formulée à travers des lois et des règlements moraux, une spectaculaire répression sexuelle s'installe dans le sillage des Églises et des États qui cherchent désormais à imposer leur ordre en contrôlant de près les corps et les âmes. La pression s'intensifie particulièrement dans les milieux citadins tentés par des philosophies du renoncement religieux et de la modération des appétits. Les bourgeoisies s'écartent de l'éthique noble de la consommation sans frein pour adopter celle de l'épargne (Max Weber), y compris dans le domaine sexuel. Cette sorte de sublimation collective alimentée par des refoulements sexuels individuels contribue, selon moi, à engendrer, ou du moins à accompagner le capitalisme et le dynamisme agressif du continent européen sur la scène mondiale. 


\section{Le rôle nouveau du mariage}

Comment le désir et les pulsions sont-ils gérés par une communauté humaine ? Ce fut essentiellement le rôle du lien matrimonial, encadré par les autorités civiles ou religieuses et divers agents locaux. De la Renaissance aux Lumières s'observe un net développement de la sphère domestique, à la suite de l'irrésistible promotion de la famille conjugale comme espace d'intimité, au détriment d'une sociabilité plus large, attachée à la paroisse, au voisinage, à l'amitié, à la parenté, aux groupes professionnels et de jeunesse. Le mouvement atteint son apogée avec le ménage bourgeois du XIXe siècle, centré sur le sanctuaire du foyer, pour s'affaiblir nettement durant le dernier tiers du XXe siècle.

Loin d'être une donnée intemporelle, le couple légitime se révèle donc étroitement dépendant de l'évolution générale de la civilisation. Du milieu du XVIe siècle à celui du XXe, le noyau conjugal classique constitua l'unité de base primordiale, le lieu de rencontre privilégié des corps individuels et des normes, le seul espace licite d'expression de la sexualité. Mariage et vie domestique encadraient très étroitement l'acte vénérien. Il fallait passer la bague au doigt pour y goûter, comme le montre le très faible taux de naissances illégitimes dès le XVIIe siècle (autour de 1\%, dans les campagnes françaises). Garçons et filles célibataires pratiquaient apparemment une grande continence durant plus d'une dizaine d'années, entre la puberté et la cérémonie nuptiale, car en l'absence de contraception efficace, des coïts fréquents auraient fait bondir les statistiques de bâtardise. Mais comment faisaient-ils pour résister aux tentations de la chair? Certains chercheurs évoquent le recours aux plaisirs solitaires, ou à des pratiques juvéniles homosexuelles, d'autres soutiennent la thèse de l'ascétisme, à l'exemple des moines et des saints.

Il est vrai que le XVIIe siècle enregistre une puissante désexualisation, qui touche même le couple légalement constitué, désormais fermement incité à exercer les devoirs conjugaux sans y chercher la volupté. Pour les moralistes, celle-ci désigne les excès charnels qui dégradent l'être humain. Elle se distingue subtilement du plaisir, que les médecins du temps estiment nécessaire, aussi bien pour la femme que pour l'homme, afin de procréer dans de bonnes conditions. Les confesseurs conseillent de ne pas aimer trop intensément son conjoint et cherchent à s'insinuer au fond des alcôves en questionnant les fidèles sur les positions adoptées ou les types de caresses. Le seul comportement admissible, prétendent-ils, est la pénétration fécondante, le mâle au-dessus de sa partenaire ( «la position du missionnaire »), sans chercher la jouissance pour elle-même. Ces prescriptions invitent à accepter une sorte de sublimation, ou du moins à modérer la sensualité, afin d'éviter la damnation. En France, après 1640, l'arme de la peur des tourments infernaux perd de l'importance.

Qu'elles soient ou non bien appliquées par ceux auxquels elles s'adressent, probablement surtout des citadins, les prescriptions religieuses s'appuient toujours sur une notion d'économie personnelle des passions. Les conduites qui en découlent se distinguent très nettement de celles des paysans, pour lesquels le refoulement des «bas instincts » durant un long célibat se trouve surtout assuré par la surveillance constante exercée à travers le regard des autres. 


\section{Un nouveau modèle sexuel à partir de 1700}

\section{Naissance d'un troisième " genre " sodomite}

Au Nord-Ouest, du nouveau ! Tandis que le reste de l'Europe, le sud en particulier, demeure très longtemps imprégné par la tradition paysanne des trois blocs (hommes adultes, femmes, jeunes hommes), Paris, Amsterdam et Londres se dotent à partir de 1700 d'un modèle sexuel différent, appelé à durer jusqu'aux années 1960. Il repose sur une représentation très neuve de la masculinité, qui conduit à la promotion du mariage conjugal dans le cadre du foyer bourgeois, ainsi qu'à des transformations des rapports d'autorité et des échanges humains.

Au siècle des Lumières, les sociétés européennes les plus dynamiques prohibent le désir homosexuel classique. Il faisait jusque-là l'objet d'une certaine compréhension, la virilité n'étant nullement mise en cause pour qui affichait une attitude active avec un jouvenceau ou un mâle passif. Il n'en va plus de même à Londres, quand s'impose l'interdit absolu de tout contact sensuel avec une minorité d'adultes efféminés, qualifiés péjorativement de mollies. Concentrés dans des lieux spécifiques, ces derniers se présentent comme un véritable troisième «genre » sodomite. Une anxiété montante à ce propos trouble alors fortement la définition et la perception du sexe fort, ce qui contribue à altérer les relations entre ses membres, particulièrement en matière d'amitié. Également marquée par le rejet du vice solitaire, la masculinité idéale exclut toute équivoque et se réoriente vers la fréquentation sans complexe des bordels et des prostituées.

Outre l'émergence précoce au XVIIIe siècle d'une minorité invertie bien visible (Foucault et beaucoup d'historiens la datent seulement du siècle suivant), les résultats les plus spectaculaires de ces mutations concernent l'expansion galopante des maladies vénériennes, ainsi que l'augmentation de l'illégitimité et des pratiques extraconjugales. Paris connaît au même moment une évolution identique, dont témoignent les patrouilles de pédérastie de la police et la fulgurante augmentation de la prostitution au siècle des Lumières.

\section{Triomphe de la modération}

La sexualité humaine ressemble à un mécanisme d'horloge, car chaque mouvement donne sens au tout. La moindre modification affectant une partie se répercute sur le reste. Tandis que certains milieux préfèrent conserver les traditions ou n'y apporter que des changements marginaux, d'autres acceptent d'adopter la culture de la culpabilité personnelle et de se conformer aux procédures inédites d'encadrement de la libido qu'elle véhicule.

Les paysans ne glissent que lentement vers les novations. Ils conservent longtemps, surtout dans certaines régions éloignées des grandes villes (Gévaudan, Quercy, Corse...), l'équilibre fondé sur la mise en tutelle du groupe des jeunes mâles par la loi de la honte communautaire et sur les vengeances «d'honneur » contre les prédateurs sexuels. À l'autre extrémité du spectre social, l'ancienne morale nobiliaire de l'excès et de la magnificence, de la consommation sans frein, continue à inspirer des 
jouisseurs qui refusent la culpabilité : Sade n'est que la partie émergée de cet univers peuplé de sybarites, également bien représenté dans l'Angleterre des Lumières, par Richard Payne Knight, l'inventeur du culte de Priape, ou encore par les adhérents du club des Dilettanti.

Les autres, généralement des citadins, plus ou moins pris dans les filets d'une sexualité réprimée, sont au fond les premiers à expérimenter la puissance d'une sublimation désormais laïcisée, et non plus typique des chrétiens les plus pieux. La pente culturelle conduit à notre époque, voire au divan des psychanalystes en cas de pathologie. Car malgré le mariage tardif, l'Ancien Régime démographique se trouve déséquilibré vers 1700-1720. La population se met à augmenter rapidement. Les contemporains prennent conscience de la gravité du problème. En 1798, Malthus lance un cri d'alarme relatif au danger de voir les ressources alimentaires devenir insuffisantes : il préconise une restriction volontaire des naissances. L'Europe occidentale entre dans une ère différente, celle de la révolution industrielle. Au cœur des immenses métropoles, qui en sont les moteurs, le décollage économique du XVIIIe siècle est précédé puis accompagné par une nouvelle conception de la sexualité. L'éthique protestante en Angleterre, la reconquête spirituelle catholique en France, l'invention de la civilité dans toute l'Europe, avaient déjà contribué à apprendre à certains les vertus de l'autocontrôle et de la continence sexuelle. Le phénomène s'impose désormais au cœur de la famille.

Ce sont précisément les valeurs organisant les relations matrimoniales qui changent de manière fondamentale. Les femmes y perdent une large part de leur liberté et sont même dépossédées par les médecins de leur droit au plaisir dans les bras d'un époux. À l'origine du changement, la chasse aux sorcières des années 15801680 contribue à développer une peur de la sexualité dévorante des femmes. L'une des principales accusations portées contre les suspectes, souvent vieilles et ménopausées, est d'avoir copulé avec des démons, pour chercher diaboliquement le pur plaisir, puisqu'elles étaient stériles. Les juges assimilent la volupté féminine débridée, hors mariage, au démoniaque, et promettent aux accusées des tourments infernaux éternels. Martelé durant plus d'un siècle, le message valorise, par opposition, l'image de la bonne épouse chrétienne, qui fait son salut et son devoir envers Dieu et les hommes en engendrant sans rechercher la jouissance pour elle-même. La voie est ainsi ouverte à l'opinion selon laquelle la femme honnête ne peut avoir qu'un intérêt modéré pour les ébats amoureux. L'idée s'impose au XIXe siècle, lorsque la morale bourgeoise victorienne (en France comme en Angleterre) établit une distinction très nette entre les épouses légitimes, chastes et obéissantes, auxquelles la médecine triomphante attribue une faible appétence pour les jeux érotiques, voire une frigidité naturelle, et les prostituées libidineuses. Une telle division des rôles féminins fonctionne à l'avantage exclusif des mâles dominants. Ils gagnent sur tous les tableaux en pratiquant le double standard de comportement : le mari modèle peut à la fois être un amant empressé et fréquenter des hétaïres sans éprouver de culpabilité. De plus, les conjointes dociles sont plus étroitement confinées au foyer et ainsi d'autant mieux soustraites à la concupiscence des compétiteurs, ce qui limite le risque et la crainte de la bâtardise. Le plaisir sensuel féminin, enfin, se trouve directement relié aux catins 
qui vendent leurs charmes, manière de le dévaloriser et de prétendre que sa quête dévalue toute femme, même si elle le prend dans le lit conjugal.

Une autre mutation cruciale affecte parallèlement les garçons célibataires. Contrairement à ce que pensait Foucault, l'encadrement de leur sexualité ne date pas de l'installation tardive d'un biopouvoir au XIXe siècle. Le processus s'amorce dès l'« enfermement » d'une petite partie d'entre eux dans les internats des collèges au XVIe siècle et s'accentue par la suite, sous l'action de divers agents sociaux. La fin de l'armée de métier en France sous Louis XIV, les efforts des Églises pour moraliser la jeunesse en Europe, le renforcement des corporations urbaines, concourent à produire un âge de la vie qui n'était pas clairement perçu comme tel jusque-là. Les « fils à marier» des royaumes de jeunesse deviennent peu à peu des adolescents, coupés graduellement ou complètement (selon les groupes sociaux) de leurs semblables, pour passer sous la tutelle directe d'adultes. Parmi eux, les héritiers des privilégiés et des citadins aisés représentent un enjeu primordial. Seuls les garçons fréquentent les établissements d'enseignement secondaire et supérieur, où leurs pulsions sexuelles doivent se couler dans des cadres imposés. Ces mondes juvéniles entièrement masculins sont surveillés de près, comme en témoigne la hantise de la masturbation dans ces établissements. Mais des exutoires traditionnels existent, en particulier la sodomie dans les internats religieux de l'Ancien Régime ou dans les universités anglaises du XIXe siècle (où le culte du sport ne diminue pas vraiment le danger, jusqu'à nos jours). À Londres, le surgissement après 1700 d'une minorité homosexuelle poussée à proclamer son autonomie et à investir un espace constitué en ghetto constitue une forme de résistance aux interdits et s'explique probablement par le besoin de compenser le déclin de l'ancienne sociabilité juvénile organisée. D'autant que les menaces de châtiment éternel ou les sévères mises en garde des pères, des maîtres, des confesseurs, ont pu contribuer à développer chez les intéressés une terreur de leur propre corps : Le Chagrin des Belges (1983) du grand romancier flamand Hugo Claus en témoigne parfaitement. Intériorisation et répression font lentement le lit d'une conscience de soi désormais analysable en termes freudiens. Le refoulement des pulsions et un sens de culpabilité personnelle plus aiguisé remplacent la loi de la honte pour tenir en lisière les jeunes mâles. Ils ont néanmoins droit à des dérivatifs. Si la fréquentation des dames honnêtes leur est en principe interdite, des cohortes de prostituées leur ouvrent les bras. Ce large exutoire n'est pas seulement toléré socialement, il est encouragé par les pères eux-mêmes, qui supervisent parfois le déniaisement de leur héritier, parce qu'il s'agit d'une preuve de virilité par excellence, la seule sensualité masculine licite ne pouvant s'exercer qu'avec des femmes.

À partir du XVIIIe siècle, les hommes mûrs et établis des vastes métropoles parviennent à contrôler de près l'ensemble des échanges sexuels, sur un mode très différent des univers paysans antérieurs. Ils limitent l'accès des garçons célibataires à la reproduction et à la jouissance charnelle par le refoulement des désirs, l'interdiction de la masturbation ou de l'homosexualité et la définition idéalisée des épouses, en leur laissant comme unique dérivatif admissible la fréquentation des filles de joie, qu'eux-mêmes sollicitent sans scrupules avant de revenir goûter les charmes de la vie conjugale. 
Le bouleversement est profond. Il correspond à une modernisation du système patriarcal, sous l'effet des transformations économiques, politiques et religieuses. On peut faire l'hypothèse que son maintien durable jusqu'aux années 1960, en dépit de fluctuations, et aussi de pathologies croissantes soignées par Freud et ses semblables, a produit des vagues successives de sublimation qui ont concouru à l'exceptionnelle poussée en avant de l'Occident. Car contrairement aux paysans qui cherchent obstinément à immobiliser les forces de changement, les sociétés urbaines industrielles veulent conquérir le globe. Moins apparent que la multiplication du nombre des habitants, un facteur de la plus haute importance tient au passage d'une économie sexuelle paysanne orientée vers la permanence et le remplacement aisé d'une classe d'âge masculine par une autre, à une configuration totalement déséquilibrée au profit des mâles mariés. Non seulement les adolescents ont désormais un accès très restreint aux plaisirs sexuels, mais ils peuvent éprouver de vives inquiétudes à propos de leur avenir dans un univers économique actif et mouvant, voire douter de la validité du contrat tacite de remplacement des générations, imposé à si haut prix par les pères. C'est pourquoi, me semble-t-il, beaucoup d'entre eux, surtout s'ils sont issus des classes laborieuses ou moyennes, sont poussés à une fuite en avant : expatriations massives vers les colonies, en particulier dans le cas de l'Angleterre ; conquêtes militaires de la France révolutionnaire et impériale ; missions d'évangélisation ; grandes explorations, etc.

\section{Conclusion}

De 1700, environ, aux années 1960, s'opère une réorganisation interne des sexualités occidentales, puissamment canalisées en fonction des quatre paradigmes de Foucault définis plus haut, mais aussi d'importantes mutations structurelles affectant les rapports entre les hommes et les femmes, ainsi que de l'émergence d'un troisième « genre " inverti.

Cette longue période débute cependant par une phase de grande liberté épicurienne, au XVIIIe siècle, qui efface les rigueurs de la période antérieure et offre aux femmes de la haute société une liberté inconnue jusque-là, en particulier dans le domaine sexuel. L'histoire hésite. À Paris, le paternalisme se dilue pendant quelques générations. Madame de Pompadour règne pendant près de vingt ans sur le cœur de Louis XV et tient un rôle politique fondamental, qu'aucune autre femme n'avait jamais obtenu avant elle. Mais cette France «féminisée » dans sa culture et son art de vivre érotisés est en complet décalage avec le pays profond. Porteuse de nombreuses et profondes nouveautés, la Révolution n'empêche pourtant pas le paternalisme de reprendre rapidement l'avantage, car les droits de l'Homme ne se vivent pas de la même manière pour les femmes.

La longue époque de glaciation morale «victorienne », du début du XIXe siècle aux ruptures des années 1960, constitue un triomphe du paradigme bourgeois. Plus anciens, plus puissants et mieux implantés dans les États protestants capitalistes du Nord-Ouest, Provinces-Unies et Angleterre, les groupes sociaux dominants qui le portent triomphent également en France, après 1789, suite à l'effondrement de la tutelle aristocratique sur le pays. Ce qui les unit est un sens de l'épargne fondamen- 
tal, pour en tirer des dividendes, au lieu de les gaspiller, à la manière des nobles prodigues. Or ce même sens de l'économie s'impose également dans leur perception de la sexualité. Les médecins du XIXe siècle définissent celle-ci comme une perte de substance dangereuse, voire mortelle, en cas d'excès. D'innombrables ouvrages présentent la masturbation comme une maladie conduisant rapidement au trépas. Et l'homme sain vieillissant doit impérativement se garder d'une fougue qui menacerait sa vie (Au moins peuvent-ils se rassurer en apprenant que les épouses honnêtes n'ont qu'un goût modéré pour la chose).

De 1960 à nos jours se développe une nouvelle approche de la sexualité occidentale. Elle s'affirme résolument hédoniste en Europe occidentale, à la différence des États-Unis où domine toujours une vision fortement paternaliste du thème, marquée par un puissant attachement au mariage hétérosexuel (malgré la reconnaissance du mariage homosexuel par la Cour suprême en 2015) et par une liberté de mœurs beaucoup moins grande. La période est cependant trop courte pour pouvoir juger s'il s'agit d'un tournant décisif à long terme ou d'une simple poussée libératrice à laquelle pourrait succéder un repli frileux.

Ce grand effort actuel de redéfinition des sensualités s'accompagne en Europe d'une autonomie érotique croissante des femmes et de la reconnaissance des droits des homosexuels. Il est assurément lié à des mouvements culturels et sociaux profonds. Le modèle traditionnel du mariage se trouve fortement ébranlé, ce qui souligne l'affaiblissement du système paternaliste traditionnel qui avait accompagné la promotion de la société industrielle. Il est possible que la crise de celle-ci, productrice de chômage massif des jeunes, ait profondément dévalué le pacte entre les générations établi voici 150 ans. Décrochée de sa relation privilégiée avec la famille, à travers le mariage, la sexualité paraît de plus en plus se lier à la sphère individuelle.

Relève-t-elle pour autant du pur hédonisme, dans une société plus narcissique qu'auparavant? L'obscur objet que constitue le plaisir érotique n'est jamais réellement déconnecté des mouvements collectifs qui affectent les groupes humains. L'évolution constatée ne serait-elle pas l'indice d'une mutation en cours vers un nouvel univers numérique postindustriel ? Et dans ce cas, l'apparente individualisation de la jouissance physique ne cacherait-elle pas l'influence de subtiles pratiques de consommation qui ont pris la relève du vieux paradigme bourgeois déclinant ? La perpétuelle incitation au désir, producteur de plaisir, dans tous les domaines, dont la sexualité, ne serait-elle pas la marque d'un post capitalisme refoulant une peur du gaspillage contraire à ses intérêts ?

\section{BIBLIOGRAPHIE}

Ouvrages cités dans le texte

- Flandrin, Jean-Louis. Le Sexe et l'Occident : évolution des attitudes et des comportements. Paris: Seuil, 1981.

- Foucault, Michel. Histoire de la sexualité, 1. La volonté de savoir. Paris: Gallimard, 1976. 
- Quaife, Geoffrey. Wanton Wenches and Wayward Wives. Peasants and illicit Sex in Early Seventeenth-Century England. Londres: Croom Helm, 1979.

- Rossiaud, Jacques. La Prostitution médiévale. Paris : Flammarion, 1988.

- Weber, Max. L'Éthique protestante et l'esprit du capitalisme. Paris: Plon, 1964 ( $1^{\text {ere }}$ éd. allemande 1905).

Bibliographie des ouvrages principaux de l'auteur

- Muchembled, Robert. Culture populaire et culture des élites dans la France moderne: XVe-XVIIIe siècle. Paris: Flammarion, 1978, 398 p.

- Dupont-Bouchat Marie-Sylvie, Frijhoff Wilhelmus Theodorus Maria, Muchembled Robert. Prophètes et sorciers dans les Pays-Bas: XVIe-XVIIIe siècle. Paris: Hachette, 1978, $366 \mathrm{p}$.

- Muchembled, Robert (présenté par). La Sorcière au village: XVe-XVIIIe siècle. Paris: Gallimard, 1979, 240 p.

- Muchembled, Robert. Les Derniers bûchers: un village de Flandre et ses sorcières sous Louis XIV. Paris: Ramsay, 1981, 277 p.

- Muchembled, Robert, Sivéry, Gérard (sous la dir.). Nos ancêtres les paysans: aspects du monde rural dans le Nord-Pas-de-Calais des origines à nos jours. Lille: C.R.D.P., 1981, 300 p.

- Muchembled, Robert. Sorcières, justice et société aux 16e et 17e siècles, Paris: Imago, 1987, $267 \mathrm{p}$.

- Muchembled, Robert. L'Invention de l'homme moderne: sensibilités, mours et comportements collectifs sous l'Ancien régime. Paris: Fayard, 1988, 513 p.

- Muchembled, Robert. La Violence au village: sociabilité et comportements populaires en Artois du XVe au XVIIe siècle. Turnhout: Brepols, 1989, 419 p.

- Muchembled, Robert. Société et mentalités dans la France moderne, XVIe-XVIIIe siècle. Paris: A. Colin, 1991, 187 p.

- Muchembled, Robert. Le Temps des supplices: de l'obéissance sous les rois absolus: XVe-XVIIIe siècle. Paris: A. Colin, 1992, 259 p.

- Muchembled, Robert (sous la direction de). Magie et sorcellerie en Europe du Moyen âge à nos jours. Paris: A. Colin, 1994, $335 \mathrm{p}$.

- Muchembled, Robert. Société, cultures et mentalités dans la France moderne: XVIe-XVIIIe siècle. Paris: A. Colin, 1994, 187 p. (Réédition du livre de 1991).

- Muchembled, Robert. Cultures et société en France: du début du XVIe siècle au milieu du XVIIe siècle. Paris: SEDES, 1995, 517 p.

- Muchembled, Robert. La Société policée: politique et politesse en France du XVIe au XXe siècle. Paris: Seuil, 1998, 364 p.

- Muchembled, Robert. Une histoire du diable, XIIe-XXe siècle. Paris: Seuil, 2000, $403 \mathrm{p}$.

- Muchembled, Robert. Le temps des supplices: de l'obéissance sous les rois absolus, XVe-XVIIIe siècle. Paris: Grand livre du mois, 2000, 259 p. (Réédition du livre de 1992).

- Muchembled, Robert. Diable! Paris: Seuil, Arte éd., 2002, 198 p. 
- Muchembled, Robert. L'Invention de la France moderne: monarchie, cultures et société: 1500-1660. Paris: A. Colin, 2002, 271 p.

- Muchembled, Robert. Passions de femmes au temps de la reine Margot: 15531615. Paris: Seuil, 2003, 277 p.

- Muchembled, Robert. L'Orgasme et l'Occident: une histoire du plaisir du XVIe siècle à nos jours. Paris: Seuil, 2005, 382 p.

- Muchembled, Robert. Une histoire de la violence: de la fin du Moyen âge à nos jours. Paris: Seuil, 2008, 498 p.

- Muchembled, Robert, Bennezon Hervé, Michel Marie-José. Histoire du grand Paris: de la Renaissance à la Révolution. Paris: Perrin, 2009, 414 p.

- Muchembled, Robert. Les Ripoux des Lumières: corruption policière et Révolution. Paris: Seuil, 2011, 570 p.

- Muchembled, Robert. Une histoire de la violence: de la fin du Moyen âge à nos jours. Paris: Points, 2012, 487 p. (Réédition du livre de 2008)

- Muchembled, Robert. Insoumises: une autre histoire des Françaises, XVIe-XXIe siècle. Paris: Autrement, 2013, 327 p.

- Muchembled, Robert. Mystérieuse madame de Pompadour. Paris: Fayard, 2014, $590 \mathrm{p}$.

- Muchembled, Robert. La Civilisation des odeurs, de la Renaissance au Premier Empire. Paris: Les Belles Lettres (à paraître).

Les traductions serbes

- Mišambled, Rober. Istorija nasilja od srednjeg veka do danas. Novi Sad: Akademska knjiga, 2015.

- Mišambled, Rober. Orgazam i Zapad. Istorija užitka od XVI veka do danas. Novi Sad: Akademska knjiga, 2016.

Rober Mišambled

\section{SEKSUALNO POTISKIVANJE: POKRETAČ NAPRETKA ZAPADA OD 1500. DO NAŠIH DANA?}

\section{Rezime}

U ovom tekstu autor istražuje razvoje i odjeke snažnog seksualnog potiskivanja koje se ustoličilo u srcu zapadne civilizacije oko polovine XVI veka i koje je stvarno ustuknulo tek od šezdesetih godina XX veka. Stvaralac osnovne napetosti između libida pojedinca i kolektivnih ideala, taj proces je tokom ovog dugog vremenskog perioda stalno razvijao snažan napor sublimacije pod kulturnim plaštovima vezanim za religiju, prosvetiteljstvo, medicinu XIX veka i kapitalističko tržište, koji su se uzastopno smenjivali. Od 1960. do naših dana razvijao se nov pristup zapadnoj seksualnosti koji svedoči o dubokim kulturnim i društvenim kretanjima. 
KLJUČNE REČI: seksualno potiskivanje, zapadna civilizacija, religija, prosvetiteljstvo, medicina, kapitalizam, kultura, društvo

\title{
SEXUAL REPRESSION: THE DRIVING FORCE OF THE WESTERN PROGRESS FROM 1500 TO OUR DAYS?
}

\begin{abstract}
Summary
In this study the author examines the development and the impact of the strong sexual repression which settled in the heart of the Western Civilization around the middle of the XVI century and which really loosened only starting from the 1960's. Within the founding tension between the libido of the person and the collective ideals, this process constantly developed during this long period, creating a strong effort of sublimation, under the different successive cultural expressions in relation to the religion, Enlightenment, medicine of the XIX century and the capitalist market. From 1960 to our days, a new approach to the Western sexuality has been developed, testifying about the profound cultural and societal movements.
\end{abstract}

KEYWORDS: Sexual repression, Western civilization, Religion, Enlightenment, Medicine, Capitalism, Culture, Society 